\title{
How Do We Keep the Living Laboratory Alive? Learning and Conflicts in Living Lab Collaboration
}

\section{Louna Hakkarainen and Sampsa Hyysalo}

\author{
" To understand the dynamics of interactive learning or" \\ knowledge creation, we need to study interaction between \\ people: what was learned, how, by whom, and at what \\ level of work and organization.
}

\author{
Reijo Miettinen (2002; tinyurl.com/ls3rgg5) \\ Professor of Adult Education
}

\begin{abstract}
Living lab environments are often promoted as a way to engage private companies, citizens, researchers, and public organizations in mutually beneficial learning. Based on an in-depth case study of a four-year living lab collaboration in gerontechnology, we agree that successful living lab development hinges on learning between the parties, yet its emergence cannot be presumed or taken for granted. Diverse competences and interests of participating actors often make technology development projects complicated and volatile. The study describes two specific challenges faced in a living lab project: i) power issues between the actors and ii) end-user reluctance to participate in the development of new technology. Despite the hardships, we suggest that the living lab environment worked as a catalyst for learning between users and developers. Nevertheless, realizing the benefits of this learning may be more challenging than is usually expected. Learning for interaction is needed before effective learning in interaction is possible.
\end{abstract}

\section{Introduction}

A living lab turns users from observed subjects to active co-creators of value, ideas, and innovative concepts - it is not only a testbed (McPhee et al., 2012; timreview.ca/ article/601). It gives an opportunity to embed complex product ideas and prototypes within an environment that closely resembles the context of the product in reallife (Pierson and Lievens, 2005; tinyurl.com/9t9sylo). This opportunity, in turn, can stimulate interactions, create institutional support for innovation, and reduce innovation failures (Pierson and Lievens, 2005; tinyurl.com/ 9t9sylo).

Previous research further suggests that a living lab methodology helps in developing more context-specific insights on development and acceptance processes, and the interaction between them especially. Living lab experiments inform us about requirements of the embedding of technology in society, and they illustrate the potential societal impacts of innovation (Ballon et al.,
2005; tinyurl.com/8hox58r). Almirall and Wareham (2008; tinyurl.com/8vwtjw2) posit that living labs offer governance and structure to user contributions; help the sensing of user insights; provide solutions to the filtering problem; create societal involvement; and can be used to promote user entrepreneurship. The living lab is seen to institutionalize the meeting place for all organizations involved, and integrate and synthesize the human, social, economic, and technological processes of innovation (Niitamo, Kulkki, Eriksson, and Hribernik, 2006; Proceedings of the 12th International Conference on Concurrent Enterprising). A human-centric innovation may emerge through the process, where technology is created and challenged in interaction with human, social, and institutional elements (Niitamo et al., 2006).

In terms of innovation research and innovation management, the research on living labs appears to be at the point where an interesting new phenomenon is charted from multiple directions, for instance, by comparing projects and experiences across living labs in dif- 


\section{Learning and Conflicts in Living Lab Collaboration}

\section{Louna Hakkarainen and Sampsa Hyysalo}

ferent countries and sectors (e.g., Leminen et al., 2012; timreview.ca/article/602), by analyzing living labs as innovation intermediaries (e.g., Katzy et al., 2012; tinyurl.com/ lvroe2d), by situating living labs in the field of user-driven innovation methodologies (e.g., Ballon et al., 2005: tinyurl.com/8hox58r; Almirall et al., 2012: timreview.ca/ article/603), by examining issues related to intellectual property rights (e.g., Pitkänen and Lehto, 2012; tinyurl.com/qjne78j), and by presenting specific cases of living lab development (e.g., Bendavid and Cassivi, 2012: tinyurl.com/kuup5rb; Bourgault, 2012: tinyurl.com/mz4aegx). A type of research that is hitherto missing in the living lab domain is an in-depth longitudinal case analysis examining some key facet, such as user-developer interaction. Such studies have become commonplace in innovation research over the past three decades (Van de Ven, 1999: tinyurl.com/n5h6xv2; Russell and Williams, 2001: tinyurl.com/nxeh3sv; Garud and Gehman, 2012: tinyurl.com/k97f6tu) and have thrown significant new light on how innovation processes play out.

The present article provides a rare overview of the results of such an in-depth longitudinal case study (Hakkarainen, 2013; tinyurl.com/l8dqpsr) of some of the key aspects of living labs: user involvement, learning, and interaction between participants (Katzy et al., 2012; tinyurl.com/lvroe2d). We follow these aspects during a fouryear living lab collaboration that took place in a Finnish nursing home, and ask:

\section{What learning occurred between participants?}

2. What were the challenges in achieving this learning?

\section{How were these challenges overcome?}

Our research draws from one of the key traditions in the detailed studies of innovation, the social shaping of technology approach (Williams and Edge, 1996: tinyurl .com/kh2oncz; MacKenzie and Wajkman, 1984: tinyurl.com/ mhbbatg), and its further development, the social learning in technological innovation approach (Williams et al., 2005: tinyurl.com/ma479bl; Stewart and Hyysalo, 2008: tinyurl.com/lox4bvp; Hyysalo, 2010: tinyurl.com/qz3ebln). Alongside other detailed longitudinal approaches to innovation, the three decades of social shaping of technology research have come to emphasize that innovations are typically long and winding journeys rather than orderly projects (Williams and Edge, 1996: tinyurl.com/ kh2oncz; Van de Ven, 1999: tinyurl.com/n5h6xv2). They are characterized by high contingency and uncertainty; in- deed, there may be a "fog" over the best possible courses of action (Russell and Williams, 2001: tinyurl.com/ nxeh3sv; Höyssä and Hyysalo, 2009; tinyurl.com/kn59mhk). Learning, particularly related to uses and user contexts, has been found to be crucial to these processes and whatever success they may have (Williams et al., 2005: tinyurl.com/ma479bl; Hyysalo, 2009: tinyurl.com/mcwgdd8), because innovation is typically an affair between multiple stakeholder groups that have different cultures, priorities, and interests towards the project (Williams and Edge, 1996; tinyurl.com/kh2oncz). Different perceptions over the appropriate form and function of new technology tend to lead to tensions and conflicts between stakeholders (Miettinen, 1998: tinyurl.com/ mre2ezj; Johnson et al., 2013: tinyurl.com/lzr5y39; Latour, 1996: tinyurl.com/mgk2ot3).

Particularly in health technology innovation, learning between developers and users has been found to be of crucial importance (Hasu, 2001: tinyurl.com/pvwp3kc; Hyppönen, 2007: tinyurl.com/od997pt; Hyysalo, 2000: tinyurl .com/kyw6pma; Hyysalo, 2010: tinyurl.com/qz3ebln). The parties typically have limited capacity to absorb information from other stakeholders due to lack of time and often required extensive background understanding. Many times, the parties find it difficult to even judge which information is relevant for them (Hyysalo, 2010; tinyurl.com/qz3ebln). It is further unclear who should invest in the learning and creation of working arrangements for interaction. In all of this, the shape of technology, uncertainties about its material realization, and the types of knowledge related to it, do matter. The net outcome is that the required learning tends to become a complex issue to master and grapple with; indeed, it is a multi-level game between stakeholders (Stewart and Hyysalo, 2008; tinyurl.com/mssxkf3).

With regard to innovation management, the longitudinal studies on innovation have come to view the orderly, controlled, and linear management models better suited for incremental new-product development projects. When initiating new product types or product categories, measures such as stage gate models act more as legitimizing devices than effective tools for management (Van de Ven, 1999: tinyurl.com/n5h6xv2; Jolivet et al., 2008: tinyurl.com/lfctg7g). In dealing with high uncertainty, periodical direction assessment and re-setting appear better suited for working towards the eventually desirable and attainable shape of technology, its business case, and social implications (Duret et al., 1999: tinyurl.com/ll4wqcx; Jolivet et al., 2008: tinyurl.com/lfctg7g). 


\section{Learning and Conflicts in Living Lab Collaboration}

Louna Hakkarainen and Sampsa Hyysalo

Our living lab research continues this line of studies of the mechanisms of learning and interaction between developers and users in real-life settings. We now proceed by first introducing the development project and the main difficulties in executing such intensive longterm collaboration. Thereafter, we present how the participants overcame these challenges and what were the most important benefits of the living lab methodology. Finally we distill a set of key messages to companies and other actors who are involved or interested in living lab collaboration, especially in the field of healthcare.

\section{Research Approach, Methods, and Data}

The data and analysis methods of our study are reported at length by Hakkarainen (2013; tinyurl.com/l8dqpsr). In short, the main bodies of data are 90 meeting memos and 16 semi-structured interviews. The project personnel, who were hired to organize the collaboration and who acted as user-side innovation intermediaries, documented nearly all the meetings held with different participant groups over the course of the four-year collaboration project. We used historiographic document analysis to track down processes of learning, tensions, and conflicts between the participants, as well as the temporality of the innovation process. The length of one memo was typically one to two A4 pages. In addition to memos, the data included project reports, plans, and marketing material. Altogether, the data included 151 different documents related to the development and use of the "smart floor" (described below). The historiographic document analysis was carried out by following the principles of source criticism and was triangulated with the analysis of the interviews in order to gain understanding of the events and to capture the multiple perspectives to the innovation process. The interviews varied from recorded and transcribed interviews of over one hour, to more informal half-hour chats during a normal workday. Open coding was used to categorize both the document and interview data on different research themes, events, methods etc. Our research covers the smart floor innovation project prior to and after living lab collaboration, as well as the intertwined phases of design and use of the system during the project.

\section{Outline of the Collaboration Project}

The origins of the smart floor system are in the Helsinki University of Technology (now Aalto University: aalto.fi), where the motion-tracking technique behind it was dis- covered in the early 1990s. Years later, a group of researchers and students created the first version of the floor monitoring system, and a startup company was founded around the concept in 2005. The idea for the gerontechnological device originally came from the user side: an innovation-oriented nursing home manager became aware of the discovery and encouraged the engineers to advance the technique into a floormonitoring system for elderly care.

The system consists of: i) a sensor foil, which is installed under the flooring material; ii) the user interface on a computer situated in the office; and iii) cell phones, which the nurses carry with them during their work shifts. The movements of the residents generate alerts, which the nurses receive through the cell phones. The system can inform the nurses about, for example, a situation where a frail elderly person is getting out of bed, entering or leaving the room, entering the toilet or occupying the toilet for an unusually long time. The alarms are tailored individually to each person.

The system reached its final form during a four-year living lab undertaking, which took place in four units of a large public nursing home from 2005 to 2009. Participants in the collaboration were the startup company, researchers from the university, project personnel who acted as user-side innovation intermediaries (Stewart and Hyysalo, 2008; tinyurl.com/lox4bvp) - management and care personnel of the nursing home, IT experts from the municipal bureau of social services and health care, and indirectly the residents of the nursing home. The funding for the project came from a municipal innovation fund and was mostly used to hire project workers at the elderly care site.

From the perspective of elderly care actors, the goal of collaboration was to develop new technology and simultaneously discover ways to utilize it. The implementation started at the end of 2007 in a pilot unit where the smart floor was installed in two rooms. Later, the system was put to use in three other units, each with around 20 residents, where the sensor foil was installed in all the rooms and public spaces. An overview of the project timeline is provided in Box 1 .

The project was realized without formal co-design methods. Information exchanges took place in regular meetings, where the project workers met the end users and the developers (i.e., the nurses and the engineers), separately. User concerns were learned through weekly 


\section{Learning and Conflicts in Living Lab Collaboration}

Louna Hakkarainen and Sampsa Hyysalo

to monthly feedback meetings with the nurses discussing how the system had been utilized, what its benefits were, how it changed the care work, and how it had affected the elderly people. This feedback was complemented by observing the daily use, which the project workers valued as the most important way to collect information for the improvement of the system. Their background as care workers helped them to make sense of the daily work. But, before events got to this point, the project had to navigate a number of serious potholes, as described in the following section.

Box 1. Project timeline

1990s Motion-tracking technique is discovered at the Helsinki School of Technology

2005 Smart floor receives an award in a business idea competition; spin-off company is founded

2006 Sensor foil is installed in the nursing home building; user collaboration begins

2007 August: User interface version 1.0

November: Use of the system begins in the pilot room

2008 April: Implementation is extended to three full units

May: User interface version 1.1

June: New alarms are added to the system

September: User interface version 2.0

2009 April: User interface version 2.2; new alarms are added to the system

May: Startup company merges with an established electronics company

Fall: Living lab project ends and the smart floor is launched

2013 Smart floor is installed in over 2000 rooms in residential care facilities, mainly in Finland

\section{Birth of the Smart Floor through Conflicts and Power Plays}

At the onset of the project, the engineers and the care professionals had strongly differing understandings of the maturity of the system and each other's roles. The company was in a hurry to launch their product, but from the user perspective, the smart floor was not even ready for the test implementation. The client - as represented by nursing home staff and project workers was frustrated with the functioning of the system and severity of its bugs, and saw the engineers as arrogant and indifferent to the welfare of the residents, whereas the company saw the users' requests as unreasonable and unrealistically scheduled. The goal of the company was to create a generic product instead of a tailored system; accordingly, the engineers were skeptical about the client's demands. A struggle for power over the project ensued. The key issues revolved around how quickly and accurately the developers had to answer to the wishes and demands of the care professionals, and who finally decided what functionalities would be developed into the system. The events culminated in the nursing home management and project workers refusing to proceed with the implementation unless their suggestions and demands were met. At the end of 2007, the conflict culminated in the resignation of several members of the living lab project, bringing the whole project to the verge of collapse.

Nevertheless, when the rollout of the system began at the beginning of 2008, the developers, project workers, and management of the nursing home found common ground for carrying forward the project. The hiring of a new project coordinator seemed to be essential for the new consensus. At this point, the innovation project manager wanted to find an independent and innovative negotiator, someone who would be able to change perspectives when needed, instead of just being a passionate advocate of the user side. They were looking for a person who could convince all the stakeholder groups of each other's good intentions and react quickly to changing circumstances, in other words, a genuine innovation intermediary. Nevertheless, this person had to be practical enough to push through the demanding implementation phase.

Pushing forward with the rollout of the system required the developers, project workers, and nursing home management to ally against the care personnel, many of whom were reluctant to use the system or participate 


\section{Learning and Conflicts in Living Lab Collaboration}

\section{Louna Hakkarainen and Sampsa Hyysalo}

in its improvement. Because of heavy and demanding work, the nursing staff was unwilling to study new things alongside their normal workload or to change their work routines. The nurses saw themselves as caregivers, not machinists, and were generally reserved about complex gerontechnological devices (tinyurl.com/ $k 5 z 7 k 2 c)$. Broader societal dissatisfaction with the financing of elderly care services also loomed in the background. Many care workers boycotted the smart floor, for instance by not carrying the cell phone with them during their shift, and continuing to work as they used to. In these circumstances, the commitment of the nursing home management to the implementation proved to be crucial. The use of the system and attendance at the feedback meetings was made obligatory for the nursing staff, yet they were given a chance to transfer to another unit. The manager of the innovation project was a former manager of the nursing home, which seemed also to play a role in building the commitment of the department managers to the living lab project and overcoming the resistance of the nursing home staff.

During the implementation, the strict discipline was counterbalanced by the devotion of the newly hired project staff, who were also care professionals by education. They spent time in the living lab units every day and helped the nurses in the implementation of the system, occasionally also in normal care duties. The distress of the nurses was discussed in the weekly feedback meetings, where the care personnel had an opportunity to speak out, comment on the system, and express new development ideas.

Unfortunately, the disgruntled care personnel were not very keen on generating development ideas. The responsibility to develop the system further was left on the shoulders of the project workers, especially the new project coordinator. As noted, the project workers observed use, identified problems and solutions with the engineers, and thought about ways to utilize different functionalities and properties of the system with the care personnel. Another important theme of discussion with the nurses was the question of how the system should be used in order to produce optimal results: for example, how to determine the right mix of alarms for each resident, how the system affects elderly people in the long term, and what should be done when a nurse receives overlapping alarms. The project workers and the care personnel also thought about the challenges the living lab project created, for example what should be done when the system does not work the way it is supposed to.
Hence, as unfortunate the tensions and conflicts were, they did "hammer in" each stakeholder group's realities and priorities to the others, thereby leading to deeper and more appreciative collaboration. Learning sensible ways to organize and time collaboration as well as learning to listen and respond to other party's concerns had to be achieved before mutually beneficial collaboration was achieved.

\section{Fruits of the Living Lab Collaboration}

Despite the challenges, the benefits of living lab collaboration for the innovation project appear formidable. Before the user collaboration, the operating idea of the system was limited to detecting instances when elderly residents accidentally fell in the nursing home environment. During the living lab project, the system evolved from a simple "fall down alarm" to a precautionary nursing tool, which instead of simply alarming the falls actually aimed to prevent them. Fall-down detection alone had relatively low value, because falls were detected fairly quickly in a nursing home environment anyway. The living lab collaboration, thus, helped the company to change the focus as well as the value promise of the system before the market launch. The falldown alarm evolved to a smart floor.

During the living lab project, several new alarms were added to the system. Moreover, unexpected uses emerged and were conveyed to the company. For instance, in case of a fall, the nurses used recorded data about the movements of the residents to diagnose potential risk factors in order to prevent new falls. Improving the quality of care, such as reducing the use of movement-restriction devices (e.g., bedside rails), was an important motivation for the municipal actors to start collaboration with the company and the university of technology in the first place. During the collaboration, the system evolved to reach that goal. The nurses also kept track of all the false alarms sent by the system, which enabled the company to fix a large element of the technical bugs before the large-scale marketing of the system began.

In summary, the living lab collaboration helped the company to redirect the focus of its product to a more valuable opportunity, gain new product features and value-added uses, and helped in weeding out bugs in the system. Equally important, the company gained a profound understanding of the use contexts and reallife benefits of their product, which included how the smart floor changes care work, what efficient implementation and use of the system require from the end 


\title{
Learning and Conflicts in Living Lab Collaboration
}

\author{
Louna Hakkarainen and Sampsa Hyysalo
}

users as well as from the company, and how the system affects the residents in the long run. During the collaboration, the company reached an in-depth understanding of the benefits, functioning, effects, implementation, risks, and possibilities of their product as well as the realities of the elderly care field in general. This knowledge helped the company to market their product and to support the implementation process in new client organizations.

\section{Key Messages Emerging from the Case}

Successful learning between developers and users can lead to a crucial yield with regard to the innovation process, but it is not an automatic feature of living lab collaboration per se. It requires often painstaking and conflict-ridden effort to establish such learning, even though the living lab setting and the commitment of parties to this collaborative mode of development may act as facilitating conditions. The case shows that, in high-dependability environments such as health and social care, particular attention should be paid to the following facets of living lab collaboration:

First, participants should chart different priorities and restrictions at the onset of collaboration: what issues the parties will be most concerned about, what issues are likely to be difficult to compromise, and what the conditions are in both work practice and in the technology that the parties can be flexible about.

Second, the participants should be prepared to handle conflicts, hire competent intermediary actors, and establish adequate governance structures in both organizations before the beginning of the collaboration. The needs of the project should be reviewed in the course of the collaboration, which might be difficult in the case of a rigid project plan. Regular meetings, face-to-face communication, and adequate ways to agree on scheduling are further issues that facilitate learning and help to build trust between the participants. We also recommend seeking adequate collaboration tools - in cases, just memos and lists can do the job, but at other times prototypes, mock-ups, and digital collaboration platforms may be needed.

Third, it is crucial to find adequate innovation intermediaries who can mediate between both developer and user contexts: relying solely on general process facilitation is unlikely to be sufficient. In the smart floor case, the intermediaries had to continuously adjust to unexpected situations and play several different roles. This task required creativity, negotiation skills, independ- ence, interest in developing technology as well as elderly care practices, and the capacity to build trust between the parties. This flexibility was made possible by a loose project plan and by the project workers' sufficient understanding of the user context through their own background in care work.

\section{Conclusion}

Most researchers see collaborative learning among stakeholders in real-life environments as the core rationale for setting up living labs. The current case analysis lends support to this view. Users, indeed, became co-creators of value, ideas, and innovative concepts (McPhee et al., 2012; timreview.ca/article/601). A complex product was successfully embedded in a demanding context (Ballon et al., 2005: tinyurl.com/8hox58r; Pierson and Lievens, 2005: tinyurl.com/9t9sylo), and in doing so, interactions and institutional support were fostered and a governance structure for user and developer contributions was created (Almirall and Wareham, 2008; tinyurl.com/8vwtjw2). Insights on development and acceptance processes, the value proposition of innovation, and on deployment processes were formed (Pierson and Lievens, 2005; tinyurl.com/9t9sylo). We dare to state, that without the living lab, the current success case would likely have been another innovation failure.

The case study, however, also shows how laborious and volatile such long-term and intensive collaborative undertaking can be. Before there was effective learning in interaction, there had to be learning for that interaction (Hyysalo, 2009: tinyurl.com/mcwgdd8; 2010: tinyurl.com/ qz3ebln). The early phases were characterized by the stakeholders' inability to understand and cater for each other's key concerns. The company staff underestimated the weaknesses of their prototype, did not take reliability issues seriously enough, and did not appreciate how superficial was their understanding of the elderly care context. The care personnel, in turn, were unwilling to learn to use and to work with a complex, incomplete system in addition to their demanding care duties.

The case provides further suggestions about what types of actions may turn the divergent interests and competences in to complementary ones. The active role of innovation intermediaries appears to be central, as does their deep-seated knowledge with regard to user practices. This central role helped them to seek innovation relevant information from daily use and to understand user concerns. Their frequent face-to-face communication with both parties and (by then) the genuine oppor- 


\section{Learning and Conflicts in Living Lab Collaboration}

Louna Hakkarainen and Sampsa Hyysalo

tunity to make a difference helped to build trust and overcome resistance. Further research on innovation intermediaries in living lab undertakings is needed in order to better support and enhance the learning processes in living labs. The nursing home management who forced system use and the company that continued its commitment to the collaboration also played key role in the success. The deepest knowledge transfer to the company came through hiring the key project intermediary (i.e., the project coordinator) upon completion of the project. The learning in collaboration succeeded without formal co-design methods or arrangements; it largely relied on the intermediaries' firsthand acquaintance of elderly care contexts. Knowledge of such means or having developer-side intermediaries to distill findings also could have been helpful.

To date, in-depth longitudinal analyses of living lab collaboration have been rare. The current case overview gives a glimpse of their merits in describing the microprocesses of living lab development, and how to come to better grips with them (Katzy et al., 2012; tinyurl.com/lvroe2d). Such research-based descriptions of practical living lab collaboration and change over time are needed to give managers, facilitators, and workers of living labs a better sense of the processes at stake. In terms of further research, such analyses can provide grounds for comparison between living lab development with projects conducted without living labs, and how this might vary in different sectors and in different kind of living labs.

\section{About the Authors}

Louna Hakkarainen, M.Soc.Sci, is a Doctoral candidate in the School of Arts, Design and Architecture at Aalto University in Helsinki, Finland. She is also finishing her licenciate degree in the University of Helsinki's Faculty of Social Sciences. Her research focuses on social shaping of technology, living lab development, and facilitation.

Sampsa Hyysalo is an Associate Professor in CoDesign in Aalto University's School of Arts, Design and Architecture, and he is a Senior Researcher at the Aalto University School of Business in Helsinki, Finland. Sampsa's research and teaching focus on user involvement in innovation and the co-evolution of technologies, practices, and organizations. He received his $\mathrm{PhD}$ in Behavioral Sciences from the University of Helsinki and holds a Docentship in information systems, specializing in user-centered design. Sampsa has published 30 peer-reviewed articles, and his most recent books are Health Technology Development and Use: From Practice-Bound Imagination to Evolving Impacts and Käyttäjä Tuotekehityksessä-Tieto, Tutkimus, Menetelmät (Users in Product Development-Knowledge, Research, Methods).

Citation: Hakkarainen, L. and S. Hyysalo. 2013. How

Do We Keep the Living Laboratory Alive? Learning and

(cc) BY

Innovation Management Review. December 2013:

16-22.

Keywords: living labs, learning, collaboration, conflicts, health care, gerontechnology 